\title{
THE EFFECTS OF TESTOSTERONE ON THE SEXUAL SKIN AND GENITALIA OF THE MALE TALAPOIN MONKEY
}

\author{
A. F. DIXSON* AND J. HERBERT $\uparrow$ \\ * Department of Anatomy, University of Birmingham, and \\ $\dagger$ Department of Anatomy, University of Cambridge
}

(Received 27th November 1973)

Adult males of a number of Old World monkeys possess a coloured 'sexual' skin, a term referring to an area of specialized skin covering the perineum, genitalia and adjacent areas. The sexual skin may be either blue, as in some Cercopithecus monkeys and in talapoins (Miopithecus talapoin), or red, as in rhesus monkeys and some baboons, or a mixture of the two colours, as in drills and mandrills (Napier \& Napier, 1967). Despite much speculation, there is still little definite experimental evidence on the function of the sexual skin, though it is likely to act as a behavioural signal during sexual or aggressive interactions (Wickler, 1967; Crook, 1972). The hormonal control of its colour is therefore of particular interest. In rhesus monkeys and hamadryas baboons, castration results in the red sexual skin fading rapidly to a pale pink, and subsequent administration of testosterone or oestradiol restores the original colour (Zuckerman \& Parkes, 1938, 1939; Vandenbergh, 1965). The absence of comparable information on the blue skins of the talapoin or on that of any Cercopithecus monkey prompted the present study.

The adult male talapoin has a dark blue sexual skin covering a triangularshaped perineal area bounded by the two ischial callosities posteriorly and the scrotum anteriorly. The skin of the scrotum itself is pale blue in some individuals but almost colourless in others. The perineal sexual skin of juvenile males is slate-grey in colour.

Six adult males were castrated and the colour of the perineal skin was measured by comparing it under standardized lighting conditions at approximately 14-day intervals with Munsell coloured chips. These are a standardized series of colours expressed in terms of hue (i.e. shade), value (i.e. lightness or darkness) and chroma (i.e. saturation). Animals were observed for at least 6 months after castration and, in one case, for 14 months.

There was no change in the colour of the sexual skin in any male even 14 months after castration. Measurements of plasma testosterone (Dixson, Herbert \& Rudd, 1973) showed very low levels (usually $<1 \mathrm{ng} / \mathrm{ml}$ ) so it seems unlikely that androgens from an alternative source, e.g. the adrenal, were responsible for maintaining the sexual skin's colour following castration.

A sample of sexual skin was taken from a castrated (untreated) and from an intact male. It was examined histologically by staining $7-\mu \mathrm{m}$ sections either by the Masson-Fontana technique or by Schmorl's ferricyanide reaction for demonstrating melanin. Both specimens showed a similar dense layer of 
melanin-like pigment situated in the dermis. This pigment is presumed to be responsible for the skin's blue colour, and contrasts with the red colour of the rhesus monkeys' sexual skin which is probably due to vascular change. The blue sexual skin of the vervet monkey ( $C$. aethiops) also contains melanin in the dermis (Machida \& Giacometti, 1967).

A juvenile male with a slate-grey sexual skin was given thrice weekly subcutaneous injections of testosterone propionate $(4.2 \mathrm{mg} /$ week for 7 weeks; $8.0 \mathrm{mg} /$ week for 4 weeks; $16.0 \mathrm{mg} /$ week for 6 weeks). A second juvenile received thrice-weekly injections of oestradiol benzoate $(15 \mu \mathrm{g} /$ week for 4 weeks). A third juvenile was given arachis oil as a control. Since the animals were feral,

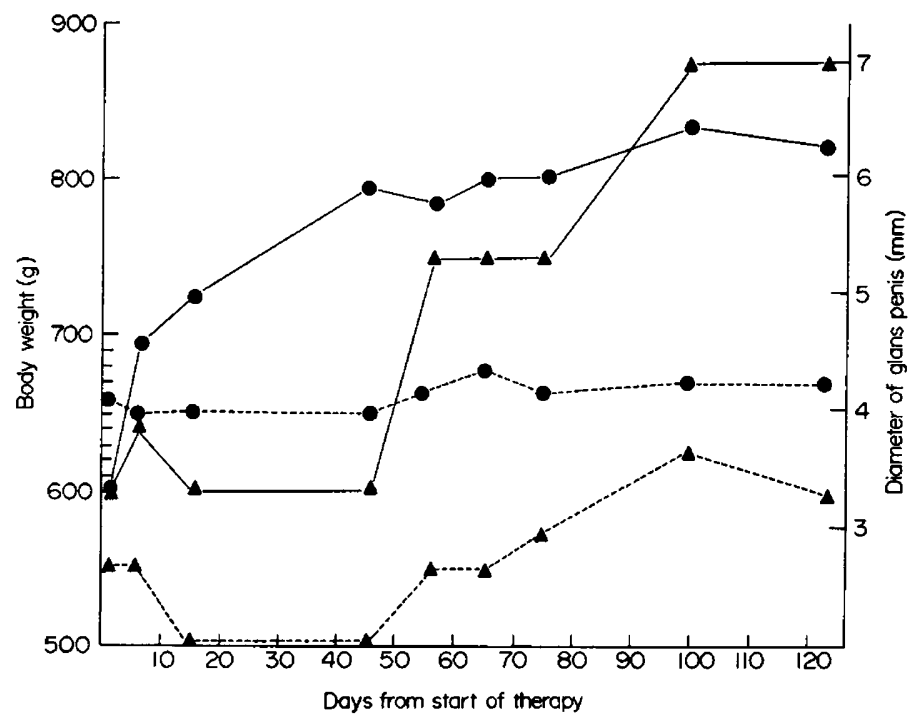

TExT-FIG. 1. The effects of testosterone upon the body weight $(\Delta)$ and diameter of the glans penis $(\bullet)$ in a juvenile talapoin monkey: -, experimental male; -- -, control male.

their ages were unknown but their initial body weights were comparable (Text-fig. 1) and about half that of the adult (A. F. Dixson and J. Herbert, unpublished observations). In contrast to some other parameters (see below), the colour of the sexual skin was unaffected by any of these treatments.

The diameter of the glans penis and that of the distal penile shaft were measured with calipers at approximately 14-day intervals, and, in adult males, the condition of the penile spines was examined under a binocular operating microscope. The dimensions of the penis showed no change after castration in the six adult talapoins, but the penile spines which covered the glans and distal shaft atrophied and became very scarce. Treating these males with testosterone (25 mg implanted subcutaneously followed in three cases by a further 75 to 100 $\mathrm{mg}$ implant) apparently restored the penile spines to their original condition. The same treatments returned blood testosterone levels to values within, or greater than, the normal range in the intact adult male talapoin (Dixson et al., 1973). Similar effects of castration on penile spines have been found in rats, 
cats and rhesus monkeys (Beach \& Levinson, 1950; Aronson \& Cooper, 1967; J. Herbert, unpublished observations).

In the juveniles, the diameter of the penis increased markedly after testosterone treatment (Text-fig. 1). The prepuce adhered firmly to the glans in untreated juveniles but became free during testosterone therapy. Furthermore, small whitish structures, possibly the opening of glands, became visible on the scrotal skin. Oestradiol caused the prepuce to become pink and swollen, and the scrotum also swelled slightly as in juvenile male rhesus monkeys given the same treatment (Zuckerman, van Wagenen \& Gardiner, 1938).

Treating the juvenile with testosterone resulted in its becoming more thickset in appearance, particularly around the chest, shoulders and thighs, and its body weight increased (Text-fig. 1).

These studies show that testicular hormones have different effects on various parts of the adult male talapoin's genitalia. The blue colour of the sexual skin does not seem to be dependent upon testosterone in either adult or juvenile (in contrast to findings in the macaque). The initial growth of the glans penis and penile shaft is markedly stimulated by testosterone, but once adult size has been attained, withdrawal of testosterone has little effect. Penile spines, on the other hand, retain their sensitivity to testosterone and its withdrawal in the adult results in degeneration of the spines.

One of us (A.F.D.) was in receipt of a postgraduate studentship from the Science Research Council.

\section{REFERENCES}

Aronson, L. R. \& Cooper, M. L. (1967) Penile spines of the cat: their endocrine-behaviour relations. Anat. Rec. 157, 71.

Beach, F. A. \& Levinson, G. (1950) The effects of androgen on the glans penis and mating behaviour in castrated male rats. $\mathcal{F}$. exp. Zool. 114, 159,168.

CROок, J. H. (1972) Sexual selection, dimorphism, and social organisation in the primates. In Sexual Selection and the Descent of Man, pp. 231-281. Ed. B. Campbell. Aldine, Chicago.

Dixson, A. F., Herbert, J. \& Rud, B. T. (1973) Gonadal hormones and behaviour in captive groups of talapoin monkeys. $\mathcal{F}$. Endocr. 57, xli.

MACHIDA, H. \& GiacometTi, L. (1967) The anatomical and histochemical properties of the skin of the external genitalia of the primates. Folia primatol. 67, 48 .

Napier, J. R. \& Napier, P. H. (1967) A Handbook of Living Primates. Academic Press, London.

VANDENBergh, J. G. (1965) Hormonal basis of the sex skin in male rhesus monkeys. Gen. $\mathcal{E}^{\circ}$ compar. Endocr. 5, 31 .

WICKLER, W. (1967) Socio-sexual signals and their intraspecific imitation among primates. In Primate Ethology, pp. 69-147. Ed. D. Morris. Weidenfeld \& Nicolson, London.

Zuckerman, S. \& Parkes, A. S. (1938) The effects of male hormone on a mature castrated male rhesus monkey. F. Anat. 72, 277.

Zuckerman, S. \& Parkes, A. S. (1939) Observations on secondary sexual characters in monkeys. 7. Endocr. 1, 430 .

Zuckerman, S., van Wagenen, S. G. \& Gardiner, R. H. (1938) The sexual skin of the rhesus monkey. Proc. zool. Soc. Lond. 108, 385. 